\title{
A Step to the Reionization Epoch
}

\author{
P. A. Shaver \\ European Southern Observatory, Karl-Schwarzschild-Str. 2, D-85748 \\ Garching bei München, Germany
}

\begin{abstract}
The reionization epoch may lie not far beyond the most distant known quasars and galaxies, and it might be detectable in a variety of ways. One possibility discussed here is to search for an all-sky spectral step produced by the redshifted HI that was present prior to reionization.
\end{abstract}

\section{The Advance to High Redshifts}

The high-redshift Universe has been opened up by a burst of discoveries and developments over the last decade. The most distant radio-loud and radio-quiet quasars now known are at $z=4.76$ (Hook et al. 2000) and $z=5.03$ (Fan et al. 2000) respectively. Galaxies have been found at even higher redshifts, including a radiogalaxy at $z=5.19$ (van Breugel et al. 1999) and a Ly $\alpha$-selected galaxy at $z=5.74$ (Hu et al. 1999).

However, it has been difficult to determine with certainty the evolution of the space density of quasars and galaxies at high redshift. In the case of quasars a decline above $z \sim 3$ is indicated by optically-selected samples (Warren et al. 1995, Schmidt et al. 1995, Kennefick et al. 1995), and a similar decline is found from radio samples (Dunlop \& Peacock 1990; Shaver et al. 1996). The small X-ray samples currently available do not show such a decline (Miyaji et al. 1999). The global star formation rate deduced from observations of Lyman break galaxies appears to be roughly constant at high redshifts (Steidel et al. 1999), and new discoveries in the $\mathrm{mm} / \mathrm{submm}$ range suggest the presence of a large population of dusty star-forming galaxies at $z \sim 3$ and perhaps higher. On the other hand, preliminary results on $\mathrm{Ly} \alpha$-emitting galaxies from $\mathrm{Hu}$ et al. (2000) indicate that their space density may decrease by a factor of six from $z \sim 4$ to $z \sim 6$, and Lanzetta et al. (1998) give an upper limit for even higher redshift galaxies. Thus, current evidence on evolution at high redshift is mixed.

Fossil evidence for the presence of luminous sources at $z>6$ abounds, in the form of heavy elements in quasar absorption systems and an IGM that was already highly ionized by $z \sim 5$. However, studies of the overall blanketing of quasar and galaxy spectra due to the intervening Ly $\alpha$ forest clouds show a rapid increase up to the highest accessible redshifts, suggesting that the reionization epoch is not far beyond.

The redshift of reionization is broadly constrained observationally. It is clearly above $z \sim 5-6$, and cannot be much above $z \sim 30$, as otherwise Thomson scattering by the ionized IGM would have damped out the fluctuations observed 
in the microwave background. Analytical and numerical estimates give $6<z_{i}<$ 15 (Gnedin 2000, and references therein).

\section{Detection of the Reionization Epoch}

Direct detection of the sources that reionized the Universe may be possible with the new 21st century observational facilities. The X-ray satellite CHANDRA is capable of detecting AGN out to very high redshifts, if they are there. NGST may detect large numbers of (mini)quasars, (sub)galaxies, star clusters, and possibly even individual supernovae. And in the mm/submm, ALMA may be able to detect the dust emission from (mini)quasars and (sub)galaxies.

A less direct approach to finding the first luminous objects in the Universe is to look for their effect on their immediate surroundings. Scattering of photons from sources before reionization may produce detectable Ly $\alpha$ halos around those sources (Loeb \& Rybicki, 1999). Stimulated radio recombination line emission may be produced by early HII regions of high emission measure (Spaans \& Norman 1997). And HI emission and absorption shells may be detectable around early quasars (Madau et al. 1997; Tozzi et al. 2000).

A completely different approach to detecting the epoch of reionization is to look for large-scale features caused directly or indirectly by the reionization process. There should be a global HI spectral feature present over the whole sky, due to redshifted $21 \mathrm{~cm}$ emission and/or absorption (Shaver et al. 1999; Tozzi et al. 2000). This is discussed in more detail below. Fluctuations should be present in this global HI signal (Madau et al. 1997; Tozzi et al. 2000), and these may be detectable with the SKA. There should also be a global Ly $\alpha$ spectral feature present over the whole sky in the near-IR (Baltz et al. 1999), possibly detectable with a large space mission at 2-3 AU. Finally, Thomson scattering from the free electrons at reionization will have produced damping, Doppler and polarization effects on the cosmic microwave background (Haiman \& Knox 2000).

\section{A Global HI Signal}

The neutral IGM just prior to reionization may have produced a copious 21-cm line signal from neutral hydrogen (in emission or absorption), a signal which would have largely disappeared following reionization. A signature ("step") in the continuum spectrum of the radio sky at $\sim 70-240 \mathrm{MHz}$ will therefore flag the reionization epoch, and may be detectable as a global signal over the whole sky.

The sharpness of this step depends on several factors, including the clumpiness of the Universe at that time and the nature of the first luminous objects. If reionization was initiated by a population of sparsely distributed quasars, the "step" would be ragged and irregular over the sky, appearing at different redshifts in different directions. In that case, individual HI concentrations may be more easily detected than a global step. But if it was caused by a vast number of stars well-distributed spatially, it may have been quite sudden and well synchronized over the sky, producing a well-defined all-sky spectral feature.

The observed differential antenna temperature across the "step" may be $\sim 0.02 \mathrm{~K}$. The limiting sensitivity for such an experiment, determined by the 
galactic and extragalactic foregrounds that dominate the system temperature, is $\Delta T_{\min } \sim T_{\text {sys }} / \sqrt{\delta \nu t}$, where $T_{\text {sys }}$ is the total system temperature, $\delta \nu$ is the bandwidth, and $t$ is the integration time. For $T_{\mathrm{sys}}=150 \mathrm{~K}$ (the coldest regions of the sky at $150 \mathrm{MHz}$ ) with a bandwidth of $5 \mathrm{MHz}$ and integration time 24 hours, $\Delta T_{\min } \sim 0.0002 \mathrm{~K}$. Thus the reionization step would be $\sim 100 \sigma$, independent of telescope size. Clearly sensitivity is not an issue, and the challenges are signal contamination, calibration and interference.

Galactic and extragalactic foregrounds are dominant at these frequencies, and can be complex, both in frequency and position. The question is whether a $0.02 \mathrm{~K}$ step can be extracted from this much stronger varying continuum. This emission is comprised of four components: galactic synchrotron emission $(\sim$ $70 \%$ at $150 \mathrm{MHz}$ ), galactic thermal (free-free) emission $(\sim 1 \%)$, the integrated emission from extragalactic sources $(\sim 27 \%)$, and the cosmic background itself.

The spectrum of the galactic nonthermal synchrotron emission is close to a featureless power law, although there are gradual variations in the spectral index with position on the sky and with frequency. The galactic thermal emission at high galactic latitudes, due to very diffuse ionized gas, has a well-defined spectral index of -2.1 . Galactic radio recombination lines occur every $1-2 \mathrm{MHz}$ at these frequencies, but line intensities are greatly diluted in the broad bands considered here and spectral lines can be identified and removed with observations of higher spectral resolution. The extragalactic component comes from many individual sources which can have complex spectra, but the overall extragalactic spectrum, an average over very many sources, should be smooth with no sharp features. Simulations that include all these contaminating effects indicate that the HI step from the reionization epoch may be detectable because of its relative sharpness. The remaining limitations are purely technical, and present an interesting challenge.

As the signal is present over the whole sky, the system temperature is dominated by the galactic and extragalactic foreground emissions, and this is a broadband (rather than line) observation, the experiment can in principle be done using small telescopes. The main technical issues are calibration and interference. The frequency response has to be calibrated to better than a few parts in $10^{5}$ over a wide frequency range. Possibilities include internal loads, or the use of astronomical sources - a very strong 'featureless' continuum source such as Cas A, or the moon.

For precise calibration, extremely stable internal broad-band noise sources may be the best option. However, calibration with an internal load must also take into account any frequency dependence of the load-coupling into the signal path. The use of astronomical calibration sources such as Cas A would eliminate that problem, but would require fairly large antennas and precise calibration of position-dependent frequency effects. The moon provides an interesting alternative (eg. Stankevich et al. 1969). As it moves, a true differencing experiment could be done at exactly the same position in the sky. All stable contaminating effects (galaxy, discrete sources, sidelobes) should be virtually identical and cancel.

An interesting aspect of a moon experiment is that the detection of the signal could in principle be done interferometrically. Because the moon occults the background signal it introduces spatial structure in the global edge signal. 
The spectrum of this interferometer signal contains the reionization step. Thus, use of the moon can convert an inherently single-dish experiment into an interferometer experiment, and this has major advantages.

Radio-frequency interference (RFI) is of course a major issue for observations at the frequencies of interest. In particular, the FM bands around $100 \mathrm{MHz}$ may be completely inaccessible for radio observations of the required accuracy (it would be most unfortunate if the reionization signal is in the corresponding redshift range of $\sim 12-15 !)$. The use of interferometers can greatly help in coping with interference, and techniques are being developed to excise RFI from astronomical observations using variability, delay and fringe rate information.

Thus, while challenging, detection of the HI step from the reionization epoch might be possible with techniques that are currently being developed.

\section{References}

Baltz E.A., Gnedin N.Y., Silk J. 1999, ApJ 493, L1, astro-ph/9707295

Dunlop J.S., Peacock J.A. 1990, MNRAS 273, L63

Fan X. et al. 2000, ApJ in press, astro-ph/9909169

Gnedin N.Y. 2000, ApJ, in press, astro-ph/9909383

Haiman Z., Knox L. 2000, in Microwave Backgrounds, eds. Oliviera-Costa \& Tegmark (ASF, San Francisco), in press, astro-ph/9902311

Hook, I.M. et al. 2000, in preparation

Hu E.M., Cowie L.L., McMahon R.G. 2000, in The Hy-Redshift Universe, eds Bunker \& van Breugel (ASP Conf. Ser.) in press, astro-ph/9911477

Hu E.M., McMahon R.G., Cowie L.L. 1999, ApJ 522, L9, astro-ph/9907079

Kennefick J.D., Djorgovski S.G., de Carvalho R.R. 1995, AJ 110, 2553

Lanzetta K.M., Yahil A., Fernandez-Soto A. 1998, AJ 116, 1066, astro-ph 9806226

Loeb A., Rybicki G.B. 1999, ApJ 524, 527, astro-ph/9902180

Madau P., Meiksin A., Rees M.J. 1997, ApJ 475, 429, astro-ph/9608010

Miyaji T., Hasinger G., Schmidt M. 1999, in Highlights of X-Ray Astronomy, in press, astro-ph/9809398

Schmidt M., Schneider D.P., Gunn J.E. 1995, AJ 110, 68

Shaver P.A., Wall J.V., Kellermann K.I., Jackson C.A., Hawkins M.R.S. 1996, Nat 384,439

Shaver P.A., Windhorst R.A., Madau P., de Bruyn A.G. 1999, A\&A 345, 390, astro-ph/9901320

Spaans M., Norman C.A. 1997, ApJ 488, 27

Stankevich K.S., Wielebinski R., Wilson R.E. 1969, Aust. J. Phys. 23, 529

Steidel C.C. et al. 1999, ApJ 519, 1, astro-ph/9811399

Tozzi P., Madau P., Meiksin A., Rees M 2000, ApJ 528, 597, astro-ph/9903139 van Breugel W. et al. 1999, in The Most Distant Radio Galaxies, eds Rottgering,

Best \& Lehnert (Amsterdam: KNAW), p. 49, astro-ph/9809186

Warren S.J., Hewett P.C., Osmer P.S. 1995, ApJ 421, 412 